\title{
Resposta da região F ionosférica observada por GPS durante os eventos SSW que ocorreram em 2006
}

Rodolfo de Jesus, Inez S. Batista, Instituto Nacional de Pesquisas Espaciais (INPE), São José dos Campos, SP, Brasil. Paulo R. Fagundes, Kavutarapu Venkatesh, Universidade do Vale do Paraíba (UNIVAP), São José dos Campos, SP, Brasil. Alessandro J. de Abreu, Instituto Tecnológico de Aeronáutica (ITA), São José dos Campos, SP, Brasil.

Copyright 2016, SBGf - Sociedade Brasileira de Geofísica

Este texto foi preparado para a apresentação no VII Simpósio Brasileiro de Geofísica, Ouro Preto, 25 a 27 de outubro de 2016. Seu conteúdo foi revisado pelo Comitê Técnico do VII SimBGf, mas não necessariamente representa a opinião da SBGf ou de seus associados. É proibida a reprodução total ou parcial deste material para propósitos comerciais sem prévia autorização da SBGf.

\section{Resumo}

The main objective of this study is to investigate the ionospheric $\mathrm{F}$ layer response induced by the Sudden Stratospheric Warming (SSW) events that occurred on 01 January - 08 February 2006 (from DOY 01 to DOY 39). In this investigation, we used observations from 7 GPS stations in the equatorial and low latitude regions in the Brazilian sector. The equatorial ionospheric anomaly (EIA) was perturbed during SSW events. The EIA was strongly suppressed from DOY 26 to DOY 29. This study also showed suppression of ionospheric irregularities (phase fluctuations) on the night of 20-21 January 2006, during SSW events.

\section{Introdução}

Quanto ao perfil de temperatura, a atmosfera neutra terrestre está dividida em troposfera $(0-10 \mathrm{~km}$ de altitude), estratosfera (10-50 km de altitude), mesosfera (50-80 km de altitude) e termosfera (acima de $80 \mathrm{~km}$ de altitude). No inverno (noite polar), o vento zonal na estratosfera apresenta uma alta intensidade por causa da zona fria de baixa pressão sobre o pólo de inverno. Este fenômeno que ocorre no vento zonal estratosférico durante o inverno é denominado de vórtice polar (uma região de alta vorticidade atmosférica) (Schoeberl e Newman, 2003). Segundo Mohanakumar (2008), uma perturbação no vórtice polar devido à propagação das ondas planetárias (intensificadas) acima da troposfera, pode induzir a geração do aquecimento estratosférico súbito (SSW - Sudden Stratospheric Warming).

A ionosfera é a região ionizada da atmosfera terrestre localizada entre aproximadamente $60 \mathrm{~km}$ e $1000 \mathrm{~km}$ de altitude. Esse meio ionizado é dividido em diferentes camadas, sendo que a camada $\mathrm{F}$ está localizada entre aproximadamente 150 e $1000 \mathrm{~km}$ de altitude. O fluxo de radiação solar na faixa espectral do extremo ultravioleta (EUV) é uma das principais fontes de ionização da camada $F$ ionosférica. Muitas observações e simulações de modelos têm mostrado que a ionosfera pode ter drásticas mudanças durante e após um evento SSW (ex: Chau et al., 2009; Goncharenko et al. 2010a, 2010b; Korenkov et al. 2012; Fagundes et al. 2015). Chau et al. (2009) relataram que a deriva vertical sobre Jicamarca teve uma variação semidiurna com grandes amplitudes com duração de vários dias durante um evento SSW de
2008. Goncharenko et al. (2010a, 2010b) relataram que no setor sul americano, durante o período do evento SSW de 2009, o TEC foi intensificado pela manhã e suprimido à tarde.

O objetivo principal deste estudo é investigar os efeitos dos eventos SSW, que ocorreram entre janeiro e fevereiro de 2006, na camada $F$ ionosférica equatorial e de baixas latitudes, utilizando dados de 7 receptores do Global Positioning System (GPS) localizados no setor brasileiro.

\section{Metodologia}

Nesta investigação foram utilizados os dados de GPS das estações de Imperatriz (IMPZ; 5,5오, 47,5O), Bom Jesus da Lapa (BOMJ; 13,2ํㅗ, 43,4을 $15,9^{\circ} \mathrm{S}, 47,9^{\circ} \mathrm{O}$ ), Uberlândia (UBER, 18,9오 $48,3^{\circ} \mathrm{O}$ ), São José dos Campos (SJC, 23,2ㅇ, 45,9O), Curitiba (PARA, 25,4오, 49, $2^{\circ} \mathrm{O}$ ), e Porto Alegre (POAL; 30,1오, $51,1^{\circ}$ O). As estações de IMPZ, BOMJ, BRAZ, UBER, PARA e POAL pertencem a Rede Brasileira de Monitoramento Contínuo dos Sinais GPS (RBMC). A estação de SJC pertence à Universidade do Vale do Paraíba (UNIVAP).

Os dados dos receptores GPS foram utilizados para calcular o conteúdo total de elétrons na vertical (vertical total electron content - VTEC) e as flutuações de fase ou taxas de variações do TEC (rate of TEC - ROT). Os valores do VTEC (das estações de IMPZ e SJC) foram utilizados para calcular o valor do $\triangle$ VTEC:

$\triangle \mathrm{VTEC}=\mathrm{VTEC}_{\text {de_cada_dia }}-\mathrm{VTEC}_{\text {med }}$

onde:

VTEC $_{\text {de_cada_dia }}$ - é o valor do VTEC de cada receptor GPS;

VTEC $_{\text {med }}$ - corresponde a média do VTEC de alguns dias calmos (índice $\mathrm{Kp}<3$ ) antes dos eventos SSW.

Sobre os valores do $\triangle$ VTEC foi realizada uma análise espectral (baseada em wavelets de Morlet) durante os eventos SSW que ocorreram em 2006.

Os dados de temperatura (na latitude de $90^{\circ} \mathrm{N}$ ) e vento zonal (na latitude de $60^{\circ} \mathrm{N}$ ) foram obtidos pelo satélite National Oceanic and Atmospheric Administration (NOAA). Estes parâmetros estratosféricos (temperatura e vento zonal) foram medidos para o nível de pressão equivalente a $10 \mathrm{hPa}$. Os valores dos índices $\mathrm{Kp}$ e F10.7 utilizados nesta investigação estão disponíveis online nos seguintes endereços:

http://ftp.gwdg.de./pub/geophys/kp-ap/tab/; 
http://omniweb.gsfc.nasa.gov/form/dx1.html.

\section{Resultados}

A Figura 1 mostra os parâmetros estratosféricos temperatura em $90^{\circ} \mathrm{N}$ a $10 \mathrm{hPa}(\sim 32 \mathrm{~km}$ de altitude) e vento zonal em $60^{\circ} \mathrm{N}$ a $10 \mathrm{hPa}$ entre os dias 01 de dezembro de 2005 e 28 de fevereiro de 2006. A Figura 1 também mostra as variações temporais dos índices F10.7 e Kp para o período entre 01 de dezembro de 2005 e 28 de fevereiro de 2006. As linhas tracejadas horizontais azuis indicam o valor de 4 e 5 no índice $\mathrm{Kp}$.

Os gráficos de contorno mostrando as variações dia-a-dia da anomalia ionosférica equatorial (AIE), sobre o setor brasileiro entre os dias 344 de 2005 e 44 de 2006, são apresentados como uma função da latitude geográfica e Universal Time (UT) na Figura 2.

A Figura 3 mostra a análise espectral baseada em wavelets realizada com os valores $\triangle$ VTEC das estações de IMPZ e SJC.

As Figuras 4a e 4b mostram as flutuações de fase (taxa de variação do TEC, TECU/min) das estações IMPZ, BRAZ e SJC.

\section{Discussão e Conclusões}
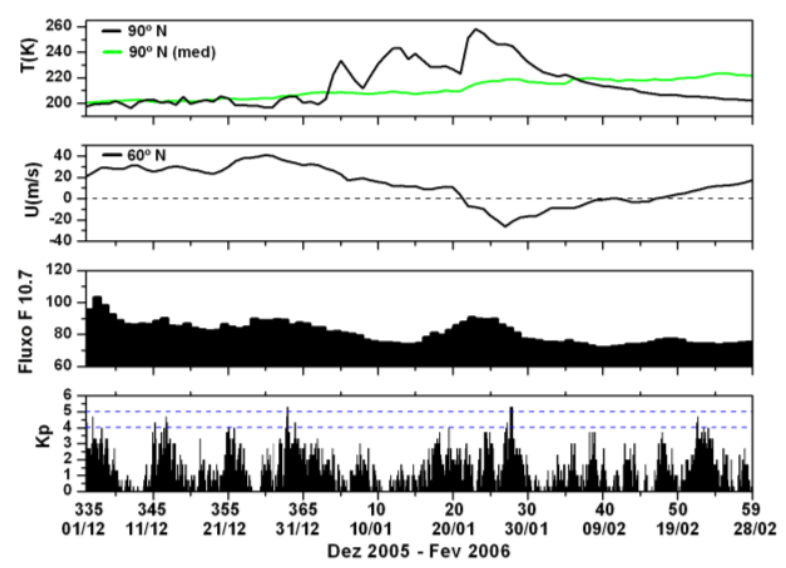

Figura 1: Variações temporais da temperatura estratosférica $\left(90^{\circ} \mathrm{N}\right.$ e $\left.10 \mathrm{hPa}\right)$, do vento zonal $\left(60^{\circ} \mathrm{N}\right.$ e $10 \mathrm{hPa}$ ), e dos índices F10.7 e Kp entre os dias 01 de dezembro de 2005 e 28 de fevereiro de 2006. No índice $\mathrm{Kp}$ as linhas tracejadas horizontais azuis correspondem aos valores 4 e 5 .

A Figura 1 mostra que entre o período de 01 de janeiro e 08 de fevereiro de 2006 ocorreram três eventos SSW no hemisfério norte. A temperatura mostra um aumento de $\sim 200 \mathrm{~K}$ (01 de janeiro) para $233 \mathrm{~K}$ (04 de janeiro) no primeiro evento. No segundo evento a temperatura exibe um aumento de $212 \mathrm{~K}$ (07 de janeiro) para $243 \mathrm{~K}$ (11 de janeiro). No terceiro evento SSW a temperatura mostra um aumento de $\sim 223 \mathrm{~K}$ (20 de janeiro) para $\sim 258 \mathrm{~K} \mathrm{(22}$ de janeiro). Como a inversão dos ventos (de leste para oeste) ocorreu apenas no terceiro evento, os dois primeiros eventos são classificados como aquecimentos de baixa intensidade e o terceiro evento como aquecimento de alta intensidade. O índice F10.7 mostra que a atividade solar estava abaixo de 90 durante os eventos SSW. O índice Kp estava abaixo de 4 e $\sim 5$ nos primeiros e terceiro eventos SSW, respectivamente.

A Figura 2 mostra uma intensa AIE nos dias 344, 345 e 346 de 2005 antes dos eventos SSW, com o pico da AIE próximo de $20^{\circ} S$ no dia 345 . Entretanto, durante os eventos SSW que ocorreram no período de 01 a 39 de 2006, a Figura 2 mostra perturbações na AIE, com uma supressão bem acentuada entre os dias 26 e 29 de 2006 no setor brasileiro. Fagundes et al. (2015) investigaram os efeitos do evento SSW de 2009 na região ionosférica equatorial, de baixas e médias latitudes no setor sul americano. Fagundes et al. (2015) relataram uma supressão na AIE durante o evento SSW de 2009. Neste trabalho os resultados da AIE (Figura 2) exibem uma boa concordância com os dados de Fagundes et al. (2015). Segundo Fagundes et al. (2015) as mudanças e supressões na AIE, durante 0 evento SSW, estão possivelmente associadas a mudanças nos campos elétricos da camada $E$ e ventos de marés na altitude da região $\mathrm{E}$.

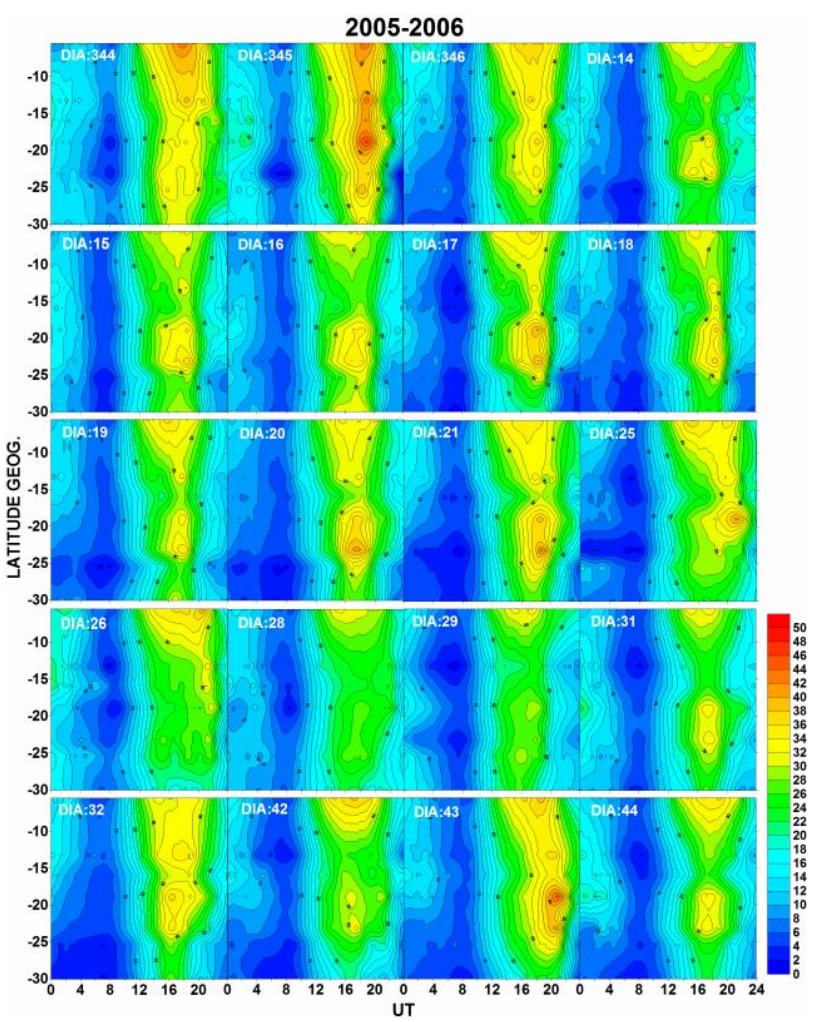

Figura 2: Gráficos de contorno mostrando as variações diárias do VTEC como uma função da latitude geográfica e UT entre os dias 344 de 2005 e 44 de 2006. 

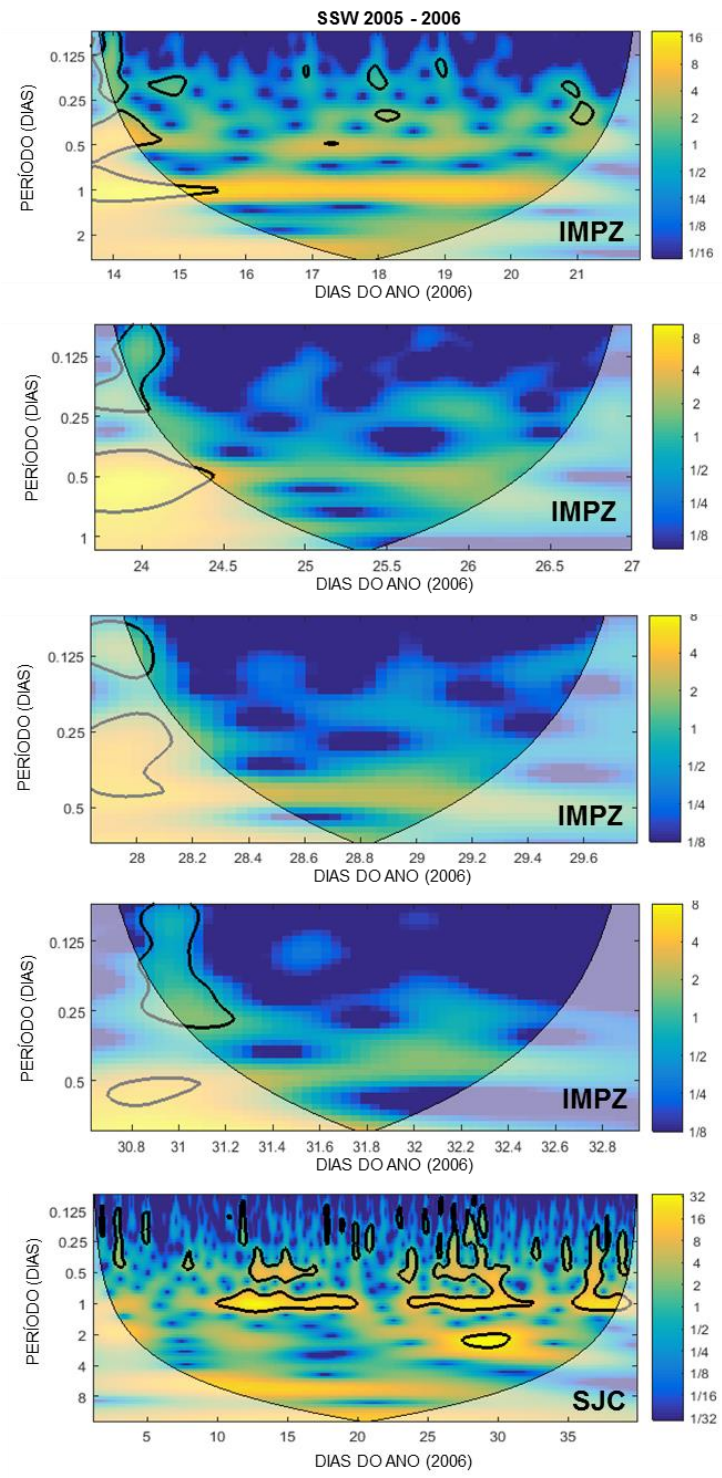

Figura 3: Wavelet para o $\triangle V T E C$ das estações IMPZ e SJC durante os eventos SSW que ocorreram em 2006.

A Figura 3 apresenta os gráficos de wavelets (do $\triangle$ VTEC) desenvolvidos para verificar as periodicidades dominantes nas estações de IMPZ (entre os dias $13 \mathrm{e}$ 33), e SJC (entre os dias 01 e 39) durante os eventos SSW de 2006. Por causa da ausência de dados de IMPZ durante alguns horários foram elaborados 4 gráficos de wavelets entre o período de 13 a 33 de 2006. A Figura 3 mostra um forte domínio de periodicidades diurnas (01 dia) entre os dias 14 e 21 em IMPZ (região equatorial). Entre o período de 16 a 32, a Figura 3 também mostra uma leve periodicidade semidiurna (0,5 dia) na estação equatorial (IMPZ). A análise espectral dos gráficos de wavelets de SJC (estação de baixa latitude localizada sob a crista sul da AIE) revelou o domínio de intensas periodicidades diurnas (01 dia) e semidiurnas (0,5 dia) entre aproximadamente os dias $12-20,25-33$ e $35-38$. As variações semidurnas na ionosfera também têm sido relatadas durante outros eventos SSW (Goncharenko et al., 2010b; Paes et al. 2014). Paes et al. (2014) relatou um comportamento semidiurno no TEC do setor brasileiro durantes os eventos SSW de 2007-2008, 2008-2009, 2009-2010 e 2010-2011. Goncharenko et al. (2010b) observou um comportamento semidiurno no TEC do setor americano, durante os eventos SSW de 2008 e 2009, associado as variações produzidas por uma mudança semidiurna na deriva vertical de íons devido a uma intensificada maré semidiurna na termosfera inferior.

As Figuras $4 \mathrm{a}$ e $4 \mathrm{~b}$ mostram antes (nas noites de 343344 e 344-345 de 2005) e após (nas noites de 41-42, 4243 e $43-44$ de 2006) os eventos SSW, respectivamente, flutuações de fase nas regiões equatoriais e de baixas latitudes. As flutuações de fase estão associadas à presença de irregularidades ionosféricas de grande escala (Aarons et al. 1997). Durante os eventos SSW que ocorreram entre os dias 01 e 39 de 2006, as Figuras 4a e $4 \mathrm{~b}$ mostram flutuações de fase em todas as noites exceto na noite de 20-21 de 2006. Sendo que nas noites de 1920 e 28-29 as Figuras 4a e 4b, respectivamente, mostram flutuações de fase apenas em IMPZ (estação equatorial). Pode-se mencionar que a Figura $4 \mathrm{~b}$ mostra na estação BRAZ ausência de dados no dia 29. De Paula et al. (2015) investigou a ocorrência das cintilações ionosféricas em SJC durante os eventos SSW de 20012002, 2002-2003 e 2012-2013. Segundo De Paula et al. (2015) os eventos SSW afetaram as cintilações ionosféricas (do tipo S4) em SJC (região de baixa latitude). Nossos resultados mostram que os eventos SSW de 2006 possivelmente afetaram as irregularidades ionosféricas (do tipo ROT) no setor brasileiro (estações equatoriais e de baixas latitudes).

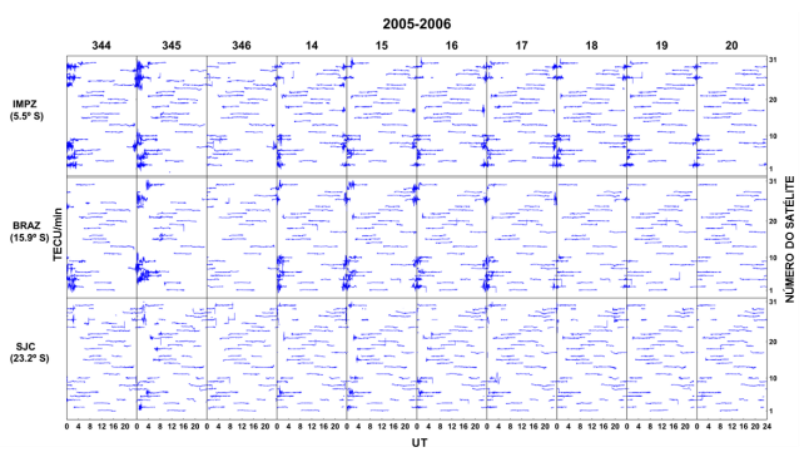

Figura 4a: Flutuações de fase ou taxas de variações do TEC (ROT) entre os dias 344 de 2005 e 20 de 2006 para as estações de IMPZ, BRAZ e SJC. 


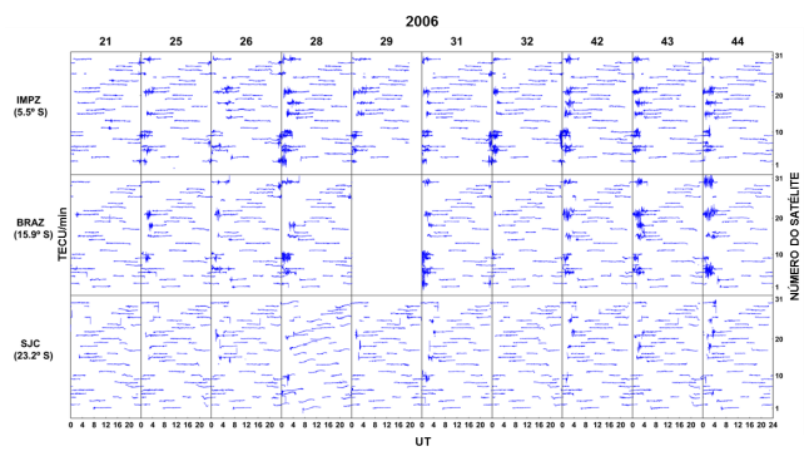

Figura 4b: Flutuações de fase (taxas de variações do TEC) obtidas por 3 estações GPS entre os dias 21 e 44 de 2006.

Concluímos que a camada $F$ ionosférica (nas regiões equatoriais e de baixas latitudes no setor brasileiro) apresentou perturbações durante os eventos SSW que ocorreram entre janeiro e fevereiro de 2006. Nos dias entre 26 e 29 de 2006 ocorreu uma forte supressão na AIE. A análise espectral do $\triangle$ VTEC (durante os eventos SSW) apresentou periodicidades de 0,5 dia e 1 dia nas estações de IMPZ (região equatorial) e SJC (região de baixa latitude), com o efeito muito mais acentuado em SJC. Durante os eventos SSW, as irregularidades ionosféricas foram observadas em todas as noites exceto na noite de 20-21.

\section{Agradecimentos}

Ao Conselho Nacional de Desenvolvimento Científico e Tecnológico (CNPq) (processo 401634/2014-0) pelo suporte financeiro. A RBMC e UNIVAP pelos dados de GPS. Ao Aslak Grinsted pelo pacote de programas wavelets disponibilizados online no endereço http://www.glaciology.net/wavelet-coherence.

\section{Referências}

AARONS J, MENDILLO M \& YANTOSCA R. 1997. GPS phase fluctuations in the equatorial region during sunspot minimum. Radio Sci., v. 32, p. 1535-1550.

CHAU JL, FEJER BG \& GONCHARENKO L. P. 2009. Quiet variability of equatorial $E \times B$ drifts during a sudden stratospheric warming event. Geophysical Research Letters, v. 36, L05101.

DE PAULA ER, JONAH OF, MORAES AO, KHERANI EA, FEJER BG, ABDU MA, MUELLA MTAH, BATISTA IS, DUTRA SLG, PAES RR. 2015. Journal of Geophysical Research, v. 120, p. 2212-2221.

GONCHARENKO LP, CHAU JL, LIU HL \& COSTER AJ. 2010a. Unexpected connections between the stratosphere and ionosphere. Geophysical Research Letters, v. 37, L10101.
GONCHARENKO LP, COSTER AJ, CHAU JL \& VALLADARES CE. 2010b. Impact of sudden stratospheric warmings on equatorial ionization anomaly. Journal of Geophysical Research, v. 115, A00G07.

FAGUNDES PR, GONCHARENKO LP, DE ABREU A J, VENKATESH K, PEZZOPANE M, DE JESUS R, GENDE $M$, COSTER AJ \& PILLAT VG. 2015. Ionospheric response to the 2009 sudden stratospheric warming over the equatorial, low, and middle latitudes in the South American sector. Journal of Geophysical Research, v. 120, p. 7889-7902.

KORENKOV YN, KLIMENKO VV, KLIMENKO MV, BESSARAB FS, KORENKOVA NA, RATOVSKY KG, CHERNIGOVSKAYA MA, SHCHERBAKOV AA, SAHAI $Y$, FAGUNDES PR, DE JESUS R, DE ABREU AJ \& CONDOR P. 2012. The global thermospheric and ionospheric response to the 2008 minor sudden stratospheric warming event. Journal of Geophysical Research, v. 117, A10309.

MOHANAKUMAR K. 2008. Stratosphere troposphere interactions an introduction. Cochin: Springer.

PAES RR, BATISTA IS, CANDIDO CMN, JONAH OF \& SANTOS PCP. 2014. Equatorial ionization anomaly variability over the Brazilian region during boreal sudden stratospheric warming events. Journal of Geophysical Research, v. 119, doi:10.1002/2014JA019968.

SCHOEBERL MR \& NEWMAN PA. 2003. Polar vortex, Encyclopedia of Atmospheric Sciences, p. 1321-1328, Elsevier, New York. 